MATHEMATICS OF COMPUTATION

Volume 70, Number 234, Pages 453-470

S 0025-5718(00)01222-9

Article electronically published on February 21, 2000

\title{
UNIFORM CONVERGENCE OF THE MULTIGRID V-CYCLE FOR AN ANISOTROPIC PROBLEM
}

\author{
JAMES H. BRAMBLE AND XUEJUN ZHANG
}

\begin{abstract}
In this paper, we consider the linear systems arising from the standard finite element discretizations of certain second order anisotropic problems with variable coefficients on a rectangle. We study the performance of a Vcycle multigrid method applied to the finite element equations. Since the usual "regularity and approximation" assumption does not hold for the anisotropic finite element problems, the standard multigrid convergence theory cannot be applied directly. In this paper, a modification of the theory of Braess and Hackbusch will be presented. We show that the V-cycle multigrid iteration with a line smoother is a uniform contraction in the energy norm. In the verification of the hypotheses in our theory, we use a weighted $L^{2}$-norm estimate for the error in the Galerkin finite element approximation and a smoothing property of the line smoothers which is proved in this paper.
\end{abstract}

\section{INTRODUCTION}

The purpose of this paper is to study the V-cycle multigrid methods for certain second order anisotropic finite element problems with variable coefficients on a rectangle. The convergence properties of the V-cycle multigrid method for second order selfadjoint elliptic finite element equations are well understood in the cases in which the differential operators are uniformly bounded and elliptic; cf. Braess and Hackbusch [3, Bramble and Pasciak [5, 6], Bramble, Pasciak, Wang and Xu [7] and the references in these papers. The common ingredient in the analysis is the so-called "regularity and approximation" condition. The success of the multigrid methods in these cases is due to the fact that the smoothers are effective in reducing the nonsmooth components of the error and the coarse grid corrections are effective in reducing the smooth components. In this paper, we shall establish a convergence theory for the standard V-cycle multigrid algorithm for anisotropic equations with variable coefficients on the unit square. We shall consider finite element approximations to this problem. For the anisotropic problem considered in this paper, the standard finite element solution has a "poor" approximation property and hence the coarse grid solves in the multigrid algorithm are not effective in reducing the smooth components of the errors. This is in contrast to the cases in which the differential operators are uniformly bounded and elliptic. When a Jacobi or a Gauss-Seidel smoother is used, the multigrid algorithm does not provide a

Received by the editor December 4, 1997 and, in revised form, June 23, 1998 and April 6, 1999. 2000 Mathematics Subject Classification. Primary 65N30; Secondary 65F10.

The work of the first author was partially supported by the National Science Foundation under grant \#DMS-9626567, and the work of the second author was partially supported by the National Science Foundation under Grant \#DMS-9805590. 
uniform reduction in the error. The remedy is to use a smoother, such as a line Jacobi or a line Gauss-Seidel smoother, that is effective in reducing components of the error in a larger part of the spectrum.

Since the usual "regularity and approximation" condition does not hold in this case, the V-cycle multigrid theory of Braess and Hackbusch [3] and Bramble and Pasciak [5] cannot be applied directly. In the case of constant coefficients, uniform convergence results for the V-cycle multigrid were established recently by Stevenson [12 15] using the multigrid theory of Hackbusch [9] and Mandel, McCormick and Bank [2, and by Neuss [11] using the multigrid theory of Bramble and Pasciak [5, 6], Xu [19] and Yserentant [20]. The main ingredient in these papers is an approximation property of the finite element spaces. This property is established in Stevenson 13 using the error estimate of Babuška and Aziz 11, and this is done by transforming the anisotropic problem to an isotropic problem by a scaling of the domain. Optimal multilevel additive preconditioners for anisotropic equations with constant coefficients were developed by Griebel and Oswald using tensor product type subspace splittings 8 . The subspace splitting is obtained by a semi-coarsening technique. Other recent related work can be found, for example, in Wittum [17 18, Stevenson [14, 16, and Hemker [10].

In this paper, we consider some anisotropic equations with variable coefficients on a rectangle. The scaling techniques which have been used for the constant coefficients case do not seem applicable to the variable coefficients problem considered here. We shall establish a uniform convergence result for multigrid V-cycle algorithms using a variant of the theory of Braess and Hackbusch. To verify the hypotheses of the theory, we shall give a direct proof of the weighted $L^{2}$-norm error estimate for the finite element approximation and a smoothing property of the line Jacobi and the line Gauss-Seidel smoothers.

Let $\Omega=(0,1)^{2}$ be the unit square, and consider the equation

$$
\left\{\begin{aligned}
\mathcal{L} u=-\left[\left(a u_{x}\right)_{x}+\left(b u_{y}\right)_{y}\right] & =f & & \text { in } \Omega, \\
u & =0 & & \text { on } \partial \Omega,
\end{aligned}\right.
$$

where, $a(x, y)$ and $b(x, y)$ are positive functions.

We are interested in the cases in which $a(x, y)$ is of unit size and $b(x, y)$ is possibly small. More precisely, we assume that $a(x, y)$ is uniformly bounded from above and below and $b(x, y)$ is bounded uniformly from above with

$$
0<a_{\min } \leq a(x, y) \leq a_{\max },
$$

and

$$
0<b(x, y) \leq b_{\max } .
$$

We do not assume, however, that $b(x, y)$ has a uniform positive lower bound.

To carry out our analysis for the multigrid algorithm, however, we shall also make the following technical assumptions on the coefficients. We assume that certain first derivatives of $a(x, y)$ and $b(x, y)$ are uniformly bounded in the following sense:

$$
\frac{|\nabla a|}{a} \leq \frac{|\nabla a|}{a_{\min }} \leq C_{a}, \quad \text { and } \quad \frac{\left|b_{y}\right|}{b} \leq C_{b} .
$$

Throughout this paper, we shall restrict our consideration to (1.1) and we shall assume that the coefficients $a(x, y)$ and $b(x, y)$ in (1.1) satisfy conditions (1.2)(1.4). 
Since $a(x, y)$ is assumed to be uniformly bounded above and below in (1.2), the first inequality in (1.4) is the same as

$$
|\nabla a| \leq C
$$

On the other hand, since $b(x, y)$ is not assumed to have a uniform positive lower bound, the second inequality in (1.4) states that that $b(x, y)$ does not change very much in the $y$ direction relative to its magnitude. This condition can also be written as

$$
\left\{\begin{array}{l}
b(x, y)=\epsilon(x) \tilde{b}(x, y) \quad \text { with } \\
\tilde{c}_{1} \leq \tilde{b}(x, y) \leq \tilde{c}_{2} \quad \text { and } \quad\left|\tilde{b}_{y}(x, y)\right| \leq \tilde{c}_{3}
\end{array}\right.
$$

Clearly, (1.5) implies the second inequality in (1.4). Conversely, if we set $\epsilon(x)=$ $\max _{y} b(x, y)$ and $\tilde{b}(x, y)=b(x, y) / \epsilon(x)$, then the second inequality in (1.4) implies that (1.5) holds with $\mathrm{e}^{-C_{b}} \leq \tilde{b}(x, y) \leq 1$ and $\left|\tilde{b}_{y}(x, y)\right| \leq C_{b}$. In our subsequent analysis, the estimate for the rate of convergence of the $\mathrm{V}$-cycle multigrid algorithm will depend on the constants in (1.2), (1.3) and (1.4), but not on a positive lower bound for $b(x, y)$. We will often use (1.5) instead of the second inequality in (1.4). Without loss of generality, we can assume $\epsilon(x) \leq 1$ in (1.5).

The weak form of (1.1) is the following: Find $u \in H_{0}^{1}(\Omega)$ such that

$$
A(u, \phi)=(f, \phi), \quad \text { for all } \phi \in H_{0}^{1}(\Omega),
$$

where

$$
A(u, v)=\int_{\Omega}\left[a(x, y) u_{x} v_{x}+b(x, y) u_{y} v_{y}\right] \mathrm{d} x \mathrm{~d} y .
$$

Here $(\cdot, \cdot)$ is the $L^{2}$ inner product. We set $\|\cdot\|_{A}=A(\cdot, \cdot)^{1 / 2}$, the "energy norm". We shall prove a uniform convergence estimate for the V-cycle multigrid algorithm for solving the finite element equations approximating (1.6).

The remainder of the paper is organized as follows. In $\$ 2$ we introduce the standard V-cycle multigrid algorithm and provide a modification of the convergence theory of Braess and Hackbusch [3]. In 33, we prove an a priori estimate for the solutions of the anisotropic problem. Standard finite element approximations to the anisotropic problem are considered in \$4 An approximation property of the Galerkin projection is proved. A weighted $L^{2}$-norm error estimate is then established by using the regularity result proved in $\$ 3$ and the duality argument of Aubin and Nitsche. The smoothing properties of the line Jacobi and the line GaussSeidel smoothers are formulated and proved in 95 , In 96 we apply the theory of 92 to the anisotropic finite element problem. It is shown that the V-cycle multigrid method with a line smoother is a uniform contraction in the energy norm $\|\cdot\|_{A}$. This convergence result is based on the approximation property of Galerkin projection and the smoothing property of the line Jacobi and the line Gauss-Seidel methods. Finally, in $\S 7$, we formulate the multigrid algorithm in terms of vectors and matrices.

\section{Multigrid ALGORITHM AND THEORY}

In this section, we consider the standard V-cycle multigrid algorithm and provide a modification of the multigrid convergence theory of Braess and Hackbusch [3]. To this end, we consider a sequence of nested finite element spaces

$$
M_{1} \subset M_{2} \subset \cdots \subset M_{J} .
$$


The finite element problem on $M_{k}$ is the following: Find $u_{k} \in M_{k}$ such that

$$
A\left(u_{k}, \phi\right)=(f, \phi), \quad \text { for all } \phi \in M_{k} .
$$

The $L^{2}$ projection $Q_{k}: L^{2} \rightarrow M_{k}$ and the Galerkin projection $P_{k}: H_{0}^{1} \rightarrow M_{k}$ are defined by

$$
\left(Q_{k} w, \phi\right)=(w, \phi), \quad \text { for all } \quad \phi \in M_{k},
$$

and

$$
A\left(P_{k} w, \phi\right)=A(w, \phi), \quad \text { for all } \quad \phi \in M_{k} .
$$

Let $A_{k}: M_{k} \rightarrow M_{k}$ be defined by

$$
\left(A_{k} w, \phi\right)=A(w, \phi), \quad \text { for all } \quad \phi \in M_{k} .
$$

Then the finite element equations can be rewritten in the form

$$
A_{k} u_{k}=f_{k}:=Q_{k} f .
$$

To define the multigrid algorithm, we need smoothing operators $R_{k}: M_{k} \rightarrow M_{k}$. We shall denote by $R_{k}^{t}$ the adjoint of $R_{k}$ with respect to the inner product $(\cdot, \cdot)$. Properties required of the smoothers will be stated later when needed.

Given an initial iterate $u^{0} \in M_{k}$, a linear multigrid algorithm produces a sequence of approximations to $u_{k}=A_{k}^{-1} f_{k}$ as

$$
u^{m+1}=\operatorname{Mg}_{k}\left(u^{m}, f_{k}\right) \equiv u^{m}+B_{k}\left(f_{k}-A_{k} u^{m}\right), \quad m=0,1, \ldots .
$$

The multigrid process $\operatorname{Mg}_{k}(\cdot, \cdot)$ (or equivalently $B_{k}$ ) is defined recursively as follows.

Algorithm 2.1. With $u^{0}$ and $g \in M_{1}$, set $\mathrm{Mg}_{1}\left(u^{0}, g\right)=A_{1}^{-1} g$ (or equivalently $\left.B_{1}=A_{1}^{-1}\right)$. For $k>1$ and $u^{0}$ and $g \in M_{k}, u^{1}=\operatorname{Mg}_{k}\left(u^{0}, g\right)$ is defined as follows:

(1) Pre-smoothing: $u^{1 / 3}=u^{0}+R_{k}^{t}\left(g-A_{k} u^{0}\right)$.

(2) Coarse grid correction:

$$
\begin{aligned}
u^{2 / 3} & =u^{1 / 3}+\operatorname{Mg}_{k-1}\left(0, Q_{k-1}\left(g-A_{k} u^{1 / 3}\right)\right) \\
& =u^{1 / 3}+B_{k-1} Q_{k-1}\left(g-A_{k} u^{1 / 3}\right) .
\end{aligned}
$$

(3) Post-smoothing: $u^{1}=u^{2 / 3}+R_{k}\left(g-A_{k} u^{2 / 3}\right)$.

To understand the multigrid algorithm, we first discuss briefly the smoothing operator, $R_{k}$. Given a smoother, $R_{k}$, the solution of $A_{k} u=f_{k}$ can be computed iteratively by the linear iteration

$$
x^{m+1}=x^{m}+R_{k}\left(f_{k}-A_{k} x^{m}\right), \quad m=0,1,2, \ldots .
$$

The error propagation operator is $K_{k}=I-R_{k} A_{k}$ and the error, $e^{m} \equiv A_{k}^{-1} f_{k}-x^{m}$, satisfies

$$
e^{m+1}=K_{k} e^{m}
$$

We assume that the above linear iteration is a contraction in the norm $\|\cdot\|_{A}$, i.e.,

$$
\left\|K_{k}\right\|_{A}=\sup _{\|v\|_{A}=\|w\|_{A}=1} A\left(K_{k} v, w\right)<1 .
$$

Set $K_{k}^{*}=\left(I-R_{k}^{t} A_{k}\right)$. Then $K_{k}^{*}$ is the adjoint of $K_{k}$ with respect to the inner product $A(\cdot, \cdot)$ and $K_{k}^{*} K_{k}$ is self-adjoint with respect to the inner product $A(\cdot, \cdot)$. Consequently

$$
\left\|K_{k}^{*} K_{k}\right\|_{A}=\left\|K_{k}^{*}\right\|_{A}^{2}=\left\|K_{k}\right\|_{A}^{2}<1 .
$$


A simple manipulation shows that

$$
A\left(K_{k} v, K_{k} v\right)=A(v, v)-\left(\bar{R}_{k} A_{k} v, A_{k} v\right),
$$

with

$$
\bar{R}_{k}=R_{k}+R_{k}^{t}-R_{k}^{t} A_{k} R_{k} .
$$

Thus, the above assumption on the smoother is equivalent to assuming that $\bar{R}_{k}$ is positive definite. Note that $\bar{R}_{k} A_{k}=I-K_{k}^{*} K_{k}$.

To estimate the rate of convergence of iteration (2.1), with $\operatorname{Mg}_{k}(\cdot, \cdot)$ defined by Algorithm 2.1 we first derive, as in Bramble and Pasciak 4, a two-level recurrence relation for the error operator of the V-cycle multigrid algorithm. Let $u=A_{k}^{-1} g$. Define $e^{0}=u-u^{0}, e^{1 / 3}=u-u^{1 / 3}, e^{2 / 3}=u-u^{2 / 3}$ and $e^{1}=u-u^{1}$. Then by the definition of the multigrid algorithm and the above discussion concerning the linear iteration (2.2), we have

$$
\begin{aligned}
e^{1 / 3} & =\left(I-R_{k}^{t} A_{k}\right) e^{0}, \\
e^{2 / 3} & =\left(I-B_{k-1} Q_{k-1} A_{k}\right) e^{1 / 3}=\left(I-B_{k-1} A_{k-1} P_{k-1}\right) e^{1 / 3}, \\
e^{1} & =\left(I-R_{k} A_{k}\right) e^{2 / 3}=\left(I-R_{k} A_{k}\right)\left(I-B_{k-1} A_{k-1} P_{k-1}\right)\left(I-R_{k}^{t} A_{k}\right) e^{0} .
\end{aligned}
$$

In the second equation, we have used the identity $Q_{k-1} A_{k}=A_{k-1} P_{k-1}$. Combining these three equations, we obtain the following recurrence relation:

$$
A\left(\left(I-B_{k} A_{k}\right) v, v\right)=A\left(\left(I-B_{k-1} A_{k-1} P_{k-1}\right) K_{k}^{*} v, K_{k}^{*} v\right), \text { for all } v \in M_{k} .
$$

Here $K_{k}=\left(I-R_{k} A_{k}\right)$ and $K_{k}^{*}=\left(I-R_{k}^{t} A_{k}\right)$ are the error propagation operators corresponding to the smoothers $R_{k}$ and $R_{k}^{t}$, respectively.

Denote by $\lambda_{k}$ the largest eigenvalue of $A_{k}$. In the standard multigrid convergence theory, the smoother, $R_{k}$, is assumed to satisfy the smoothing property

$$
\frac{\omega}{\lambda_{k}}(v, v) \leq\left(\bar{R}_{k} v, v\right), \quad \text { for all } \quad v \in M_{k}
$$

In addition, the following type of "regularity and approximation" condition is used: there exist $\alpha \in(0,1]$ and $C>0$, independent of $k$, such that

$$
\left(A_{k}^{1-\alpha}\left(I-P_{k-1}\right) v,\left(I-P_{k-1}\right) v\right) \leq C \lambda_{k}^{-\alpha} A\left(\left(I-P_{k-1}\right) v, v\right), \quad \text { for all } \quad v \in M_{k} \text {. }
$$

This type of regularity and approximation condition, however, does not hold for the anisotropic problem, and therefore, we cannot directly apply the theory of Braess and Hackbusch [3] and Bramble and Pasciak [4, 5]. We shall provide a modification of the theory of Braess and Hackbusch.

The next two lemmas are generalizations of the standard "regularity and approximation" condition for $\alpha=1$. We first consider symmetric smoothers.

Lemma 2.1. Assume that $R_{k}$ is symmetric and that there is a constant $\theta<1$ such that the spectrum $\sigma\left(K_{k}\right) \subset[-\theta, 1)$ for all $k$. Assume further that there is a constant $C_{M}$ independent of $k$ such that

$$
\left(R_{k}^{-1}\left(I-P_{k-1}\right) v,\left(I-P_{k-1}\right) v\right) \leq C_{M} A\left(\left(I-P_{k-1}\right) v, v\right), \text { for all } v \in M_{k} .
$$

Then the multigrid algorithm defined in Algorithm 2.1 satisfies

$$
0 \leq A\left(\left(I-B_{k} A_{k}\right) v, v\right) \leq \delta A(v, v), \quad \text { for all } \quad v \in M_{k},
$$

with $\delta=\gamma C_{M} /\left(1+\gamma C_{M}\right)$, where $\gamma=\max \left\{\frac{1}{2}, \frac{\theta^{2}}{1-\theta}\right\}$. 
Proof. We will prove by induction that this estimate holds. Clearly the assertion holds for $k=1$. Suppose now that the assertion holds for $k-1$, i.e.,

$$
0 \leq A\left(\left(I-B_{k-1} A_{k-1}\right) v, v\right) \leq \delta A(v, v) .
$$

Using the recurrence relation ([2.3), we have, for $v \in M_{k}$,

$$
\begin{aligned}
A\left(\left(I-B_{k} A_{k}\right) v, v\right)= & A\left(\left(I-P_{k-1}\right) K_{k}^{*} v, K_{k}^{*} v\right) \\
& +A\left(\left(I-B_{k-1} A_{k-1}\right) P_{k-1} K_{k}^{*} v, P_{k-1} K_{k}^{*} v\right) .
\end{aligned}
$$

It is straightforward to show that the induction hypothesis (2.5) implies that

$$
0 \leq A\left(\left(I-B_{k} A_{k}\right) v, v\right) \leq(1-\delta) A\left(\left(I-P_{k-1}\right) K_{k}^{*} v, K_{k}^{*} v\right)+\delta A\left(K_{k}^{*} v, K_{k}^{*} v\right)
$$

We now estimate the first term on the right hand side of (2.6). By the CauchySchwarz inequality and the hypothesis (2.4), we have, for $w \in M_{k}$,

$$
\begin{aligned}
A\left(\left(I-P_{k-1}\right) w, w\right) & \leq\left(R_{k}^{-1}\left(I-P_{k-1}\right) w,\left(I-P_{k-1}\right) w\right)^{1 / 2}\left(R_{k} A_{k} w, A_{k} w\right)^{1 / 2} \\
& \leq C_{M}^{1 / 2} A\left(\left(I-P_{k-1}\right) w, w\right)^{1 / 2}\left(R_{k} A_{k} w, A_{k} w\right)^{1 / 2} .
\end{aligned}
$$

Cancelling the common factor, we get

$$
A\left(\left(I-P_{k-1}\right) w, w\right) \leq C_{M}\left(R_{k} A_{k} w, A_{k} w\right), \quad \text { for all } \quad w \in M_{k} .
$$

Since $R_{k}$ is symmetric, $K_{k}^{*}=K_{k}$. Applying the above inequality with $w=K_{k} v$ and recalling that $R_{k} A_{k}=I-K_{k}$ and that $\sigma\left(K_{k}\right) \subset[-\theta, 1)$, we obtain

$$
\begin{aligned}
A\left(\left(I-P_{k-1}\right) K_{k} v, K_{k} v\right) & \leq C_{M}\left(R_{k} A_{k} K_{k} v, A_{k} K_{k} v\right) \\
& =C_{M}\left(\left(I-K_{k}\right) K_{k} v, A_{k} K_{k} v\right) \\
& \leq\left(\gamma C_{M}\right)\left[A(v, v)-A\left(K_{k} v, K_{k} v\right)\right] .
\end{aligned}
$$

Combining (2.6) and (2.7), we obtain

$$
\begin{aligned}
A\left(\left(I-B_{k} A_{k}\right) v, v\right) & \leq(1-\delta) A\left(\left(I-P_{k-1}\right) K_{k} v, K_{k} v\right)+\delta A\left(K_{k} v, K_{k} v\right) \\
& \leq\left(\gamma C_{M}\right)(1-\delta)\left[A(v, v)-A\left(K_{k} v, K_{k} v\right)\right]+\delta A\left(K_{k} v, K_{k} v\right) \\
& \leq \delta A(v, v),
\end{aligned}
$$

with $\delta=\gamma C_{M} /\left(1+\gamma C_{M}\right)$.

Lemma 2.1 can be modified to allow the use of nonsymmetric smoothing operators such as the line Gauss-Seidel smoother. Recall that $\bar{R}_{k}=R_{k}+R_{k}^{t}-R_{k}^{t} A_{k} R_{k}$.

Lemma 2.2. Assume that $\left\|K_{k}\right\|_{A} \equiv\left\|I-R_{k} A_{k}\right\|_{A}<1$ and that there is a constant $C_{M}$ independent of $k$ such that

$$
\left(\bar{R}_{k}^{-1}\left(I-P_{k-1}\right) v,\left(I-P_{k-1}\right) v\right) \leq C_{M} A\left(\left(I-P_{k-1}\right) v, v\right), \text { for all } v \in M_{k} .
$$

Then the multigrid algorithm defined in Algorithm 2.1 satisfies

$$
0 \leq A\left(\left(I-B_{k} A_{k}\right) v, v\right) \leq \delta A(v, v), \quad \text { for all } v \in M_{k},
$$

with $\delta=C_{M} /\left(1+C_{M}\right)$.

Proof. The proof is similar to that of Lemma 2.1 We only give an outline here. As in the proof of Lemma 2.1 the assumption in (2.8) implies that

$$
A\left(\left(I-P_{k-1}\right) w, w\right) \leq C_{M}\left(\bar{R}_{k} A_{k} w, A_{k} w\right), \quad \text { for all } \quad w \in M_{k} .
$$


Applying the above inequality with $w=K_{k}^{*} v$ and recalling that $\bar{R}_{k} A_{k}=I-K_{k}^{*} K_{k}$ and that $\sigma\left(K_{k}^{*} K_{k}\right) \subset[0,1)$, we obtain

$$
\begin{aligned}
A\left(\left(I-P_{k-1}\right) K_{k}^{*} v, K_{k}^{*} v\right) & \leq C_{M}\left(\bar{R}_{k} A_{k} K_{k}^{*} v, A_{k} K_{k}^{*} v\right) \\
& =C_{M}\left(\left(I-K_{k}^{*} K_{k}\right) K_{k}^{*} v, A_{k} K_{k}^{*} v\right) \\
& \leq C_{M}\left[A(v, v)-A\left(K_{k}^{*} v, K_{k}^{*} v\right)\right] .
\end{aligned}
$$

The rest of the proof is identical to that of Lemma 2.1

Remark 2.1. It is not necessary to solve the coarsest grid problem exactly. If the approximate coarsest grid solution satisfies $0 \leq A\left(\left(I-B_{1} A_{1}\right) \phi, \phi\right) \leq \delta_{0}<1$, for all $\phi \in M_{1}$, then Lemma 2.1 holds with $\delta=\max \left(\delta_{0}, \gamma C_{M} /\left(1+\gamma C_{M}\right)\right)$ and Lemma 2.2 holds with $\delta=\max \left(\delta_{0}, C_{M} /\left(1+C_{M}\right)\right)$.

Lemmas 2.1 and 2.2 are "soft". To apply these lemmas to Problem (1.1), we need to establish (2.4) or (2.8) with $C_{M}$ independent of $k$. For example, (2.4) can be proved, as we will see in Theorem 6.1 in Section 6 , by combining the approximation property (cf. Lemma 4.3)

$$
\left(b\left(I-P_{k-1}\right) v,\left(I-P_{k-1}\right) v\right) \leq C h_{k}^{2} A\left(\left(I-P_{k-1}\right) v, v\right)
$$

and the following smoothing property of the line Jacobi smoother (cf. Lemma 5.1):

$$
\left(R_{k}^{-1} v, v\right) \leq C\left[A(v, v)+h_{k}^{-2}(b v, v)\right] .
$$

Here $h_{k}$ is the mesh parameter. To establish (2.8) for a nonsymmetric smoother such as the line Gauss-Seidel smoother, we replace $R_{k}$ in the above inequality by $\bar{R}_{k}$. These properties will be proved later.

\section{A Regularity estimate}

In this section, we derive an a priori estimate for the solutions of the anisotropic equation (1.1). This result will be used in the next section to derive error estimates of the Galerkin finite element approximation in the energy norm and a weighted $L^{2}$-norm.

We first note that if $a(x, y)=1$ and $b(x, y)=\epsilon$ is a constant, then, by integration by parts, we have for $u \in H_{0}^{1}(\Omega) \cap H^{2}(\Omega)$

$$
\begin{aligned}
\int_{\Omega}\left(u_{x x}^{2}+2 u_{x y}^{2}+\epsilon u_{y y}^{2}\right) \mathrm{d} x \mathrm{~d} y & =\int_{\Omega}\left(u_{x x}^{2}+2 u_{x x} u_{y y}+\epsilon u_{y y}^{2}\right) \mathrm{d} x \mathrm{~d} y \\
& \leq \int_{\Omega} \frac{1}{\epsilon}|\mathcal{L} u|^{2} \mathrm{~d} x \mathrm{~d} y .
\end{aligned}
$$

The following lemma is a generalization of this fact to some variable coefficient cases.

Lemma 3.1. Let the coefficients $a(x, y)$ and $b(x, y)$ satisfy (1.2)-(1.4). Then the following a priori estimate holds:

$$
\int_{\Omega}\left(u_{x x}^{2}+2 u_{x y}^{2}+b u_{y y}^{2}\right) \mathrm{d} x \mathrm{~d} y \leq C \int_{\Omega} \frac{1}{b}|\mathcal{L} u|^{2} \mathrm{~d} x \mathrm{~d} y .
$$


Proof. Integrating by parts gives

$$
\begin{aligned}
& 2 \int_{\Omega} \frac{1}{b}\left(a u_{x}\right)_{x}\left(b u_{y}\right)_{y} \mathrm{~d} x \mathrm{~d} y \\
&=2 \int_{\Omega}\left(\left(a u_{x}\right)_{x} u_{y y}+\left(a u_{x}\right)_{x} \frac{b_{y}}{b} u_{y}\right) \mathrm{d} x \mathrm{~d} y \\
&=2 \int_{\Omega}\left(\left(a u_{x}\right)_{y} u_{x y}+\left(a u_{x}\right)_{x} \frac{b_{y}}{b} u_{y}\right) \mathrm{d} x \mathrm{~d} y \\
&=2 \int_{\Omega}\left(a u_{x y}^{2}+\left(a_{y} u_{x}\right) u_{x y}+\left(a u_{x}\right)_{x} \frac{b_{y}}{b} u_{y}\right) \mathrm{d} x \mathrm{~d} y \\
& \geq \int_{\Omega}\left\{2 a u_{x y}^{2}-\left(\alpha a u_{x y}^{2}+\frac{a_{y}^{2}}{\alpha a} u_{x}^{2}\right)-\left[\frac{\beta}{b}\left(a u_{x}\right)_{x}^{2}+\frac{b}{\beta}\left(\frac{b_{y}}{b} u_{y}\right)^{2}\right]\right\} \mathrm{d} x \mathrm{~d} y \\
&=\int_{\Omega}\left(-\frac{\beta}{b}\left(a u_{x}\right)_{x}^{2}+(2-\alpha) a u_{x y}^{2}-\frac{a_{y}^{2}}{\alpha a} u_{x}^{2}-\frac{b_{y}^{2}}{\beta b} u_{y}^{2}\right) \mathrm{d} x \mathrm{~d} y,
\end{aligned}
$$

where $\alpha$ and $\beta$ are arbitrary positive constants. As a consequence, we have

$$
\begin{array}{rl}
\int_{\Omega} \frac{1}{b}|\mathcal{L} u|^{2} \mathrm{~d} & x \mathrm{~d} y=\int_{\Omega}\left(\frac{1}{b}\left(a u_{x}\right)_{x}^{2}+\frac{2}{b}\left(a u_{x}\right)_{x}\left(b u_{y}\right)_{y}+\frac{1}{b}\left(b u_{y}\right)_{y}^{2}\right) \mathrm{d} x \mathrm{~d} y \\
\geq \int_{\Omega}\left(\frac{1-\beta}{b}\left(a u_{x}\right)_{x}^{2}+(2-\alpha) a u_{x y}^{2}+\frac{1}{b}\left(b u_{y}\right)_{y}^{2}-\frac{a_{y}^{2}}{\alpha a} u_{x}^{2}-\frac{b_{y}^{2}}{\beta b} u_{y}^{2}\right) \mathrm{d} x \mathrm{~d} y .
\end{array}
$$

Let $\gamma=\min \left(1,1 / b_{\max }\right) \leq 1$. Then

$$
\begin{aligned}
\int_{\Omega} \frac{1}{b}\left(a u_{x}\right)_{x}^{2} \mathrm{~d} x \mathrm{~d} y & \geq \gamma \int_{\Omega}\left(a u_{x}\right)_{x}^{2} \mathrm{~d} x \mathrm{~d} y \\
& =\gamma \int_{\Omega}\left(a u_{x x}+a_{x} u_{x}\right)^{2} \mathrm{~d} x \mathrm{~d} y \\
& \geq \gamma \int_{\Omega}\left(\frac{1}{2} a^{2} u_{x x}^{2}-a_{x}^{2} u_{x}^{2}\right) \mathrm{d} x \mathrm{~d} y
\end{aligned}
$$

and

$$
\begin{aligned}
\int_{\Omega} \frac{1}{b}\left(b u_{y}\right)_{y}^{2} \mathrm{~d} x \mathrm{~d} y & =\int_{\Omega} \frac{1}{b}\left(b u_{y y}+b_{y} u_{y}\right)^{2} \mathrm{~d} x \mathrm{~d} y \\
& \geq \int_{\Omega}\left(\frac{b}{2} u_{y y}^{2}-\frac{b_{y}^{2}}{b} u_{y}^{2}\right) \mathrm{d} x \mathrm{~d} y .
\end{aligned}
$$

Combining the above estimates, we obtain

$$
\begin{aligned}
\int_{\Omega} \frac{1}{b}(\mathcal{L} u)^{2} \mathrm{~d} x \mathrm{~d} y \geq(1-\beta) \gamma \int_{\Omega}\left(\frac{1}{2} a^{2} u_{x x}^{2}-a_{x}^{2} u_{x}^{2}\right) \mathrm{d} x \mathrm{~d} y+(2-\alpha) \int_{\Omega} a u_{x y}^{2} \mathrm{~d} x \mathrm{~d} y \\
+\int_{\Omega}\left(\frac{b}{2} u_{y y}^{2}-\frac{b_{y}^{2}}{b} u_{y}^{2}\right) \mathrm{d} x \mathrm{~d} y-\int_{\Omega}\left(\frac{a_{y}^{2}}{\alpha a} u_{x}^{2}+\frac{b_{y}^{2}}{\beta b} u_{y}^{2}\right) \mathrm{d} x \mathrm{~d} y \\
=\int_{\Omega}\left(\frac{1}{2}(1-\beta) \gamma a^{2} u_{x x}^{2}+(2-\alpha) a u_{x y}^{2}+\left(\frac{b}{2} u_{y y}^{2}\right)\right) \mathrm{d} x \mathrm{~d} y \\
-\int_{\Omega}\left((1-\beta) \gamma a_{x}^{2}+\frac{a_{y}^{2}}{\alpha a}\right) u_{x}^{2} \mathrm{~d} x \mathrm{~d} y-\int_{\Omega}\left(\frac{b_{y}^{2}}{b}+\frac{b_{y}^{2}}{\beta b}\right) u_{y}^{2} \mathrm{~d} x \mathrm{~d} y .
\end{aligned}
$$


Therefore

$$
\begin{aligned}
\int_{\Omega}\left(u_{x x}^{2}+\right. & \left.2 u_{x y}^{2}+b u_{y y}^{2}\right) \mathrm{d} x \mathrm{~d} y \\
\leq & C \int_{\Omega}\left[\frac{1}{2}(1-\beta) \gamma a^{2} u_{x x}^{2}+(2-\alpha) a u_{x y}^{2}+\left(\frac{b}{2} u_{y y}^{2}\right)\right] \mathrm{d} x \mathrm{~d} y \\
\leq & C \int_{\Omega} \frac{1}{b}(\mathcal{L} u)^{2} \mathrm{~d} x \mathrm{~d} y \\
& +C \int_{\Omega}\left[\left((1-\beta) \gamma a_{x}^{2}+\frac{a_{y}^{2}}{\alpha a}\right) u_{x}^{2}+\left(\frac{b_{y}^{2}}{b}+\frac{b_{y}^{2}}{\beta b}\right) u_{y}^{2}\right] \mathrm{d} x \mathrm{~d} y \\
\leq & C \int_{\Omega} \frac{1}{b}(\mathcal{L} u)^{2} \mathrm{~d} x \mathrm{~d} y+C \int_{\Omega}\left(a u_{x}^{2}+b u_{y}^{2}\right) \mathrm{d} x \mathrm{~d} y .
\end{aligned}
$$

Using the elementary inequality

$$
\int_{\Omega} u^{2} \mathrm{~d} x \mathrm{~d} y \leq \int_{\Omega} u_{x}^{2} \mathrm{~d} x \mathrm{~d} y \leq C \int_{\Omega}\left(a u_{x}^{2}+b u_{y}^{2}\right) \mathrm{d} x \mathrm{~d} y
$$

the second integral in (3.1) can be bounded as follows:

$$
\begin{aligned}
\int_{\Omega}\left(a u_{x}^{2}+b u_{y}^{2}\right) \mathrm{d} x \mathrm{~d} y & =\int_{\Omega} u(\mathcal{L} u) \mathrm{d} x \mathrm{~d} y \leq\left(\int_{\Omega} u^{2} \mathrm{~d} x \mathrm{~d} y\right)^{1 / 2}\left(\int_{\Omega}(\mathcal{L} u)^{2} \mathrm{~d} x \mathrm{~d} y\right)^{1 / 2} \\
& \leq C\left(\int_{\Omega}\left(a u_{x}^{2}+b u_{y}^{2}\right) \mathrm{d} x \mathrm{~d} y\right)^{1 / 2}\left(\int_{\Omega}(\mathcal{L} u)^{2} \mathrm{~d} x \mathrm{~d} y\right)^{1 / 2} .
\end{aligned}
$$

Cancelling the common factor and then squaring, we obtain

$$
\int_{\Omega}\left(a u_{x}^{2}+b u_{y}^{2}\right) \mathrm{d} x \mathrm{~d} y \leq C \int_{\Omega}(\mathcal{L} u)^{2} \mathrm{~d} x \mathrm{~d} y \leq C \int_{\Omega} \frac{1}{b}(\mathcal{L} u)^{2} \mathrm{~d} x \mathrm{~d} y .
$$

The lemma now follows from (3.1) and (3.2).

\section{Finite element approximation}

Let the domain $\Omega=(0,1)^{2}$ be partitioned into squares with vertices $(i h, j h), h=$ $1 / n$. We consider the linear or the bilinear finite element space $M_{h}$ associated with this partition. The Galerkin finite element projection $P_{h}: H_{0}^{1}(\Omega) \rightarrow M_{h}$ is defined by

$$
A\left(P_{h} v, \phi\right)=A(v, \phi), \quad \text { for all } \quad \phi \in M_{h} .
$$

We need the following results in proving an approximation property of the finite element solutions.

Lemma 4.1. Let $\mathcal{D}=\left(0, h_{1}\right) \times\left(0, h_{2}\right)$, and let $E$ be a side of the rectangular region D. If $v \in H^{1}(\mathcal{D})$ and $\int_{E} v \mathrm{~d} s=0$, then

$$
\|v\|_{L^{2}(\mathcal{D})}^{2} \leq\left(h_{1}^{2}\left\|v_{x}\right\|_{L^{2}(\mathcal{D})}^{2}+h_{2}^{2}\left\|v_{y}\right\|_{L^{2}(\mathcal{D})}^{2}\right) .
$$

Suppose that $b(x, y)=\epsilon(x) \tilde{b}(x, y)$ with $\tilde{c}_{1} \leq \tilde{b}(x, y) \leq \tilde{c}_{2}$, and $\epsilon(x) \leq 1$. Denote by $E$ a vertical edge of $\mathcal{D}$. If $v \in H^{1}(\mathcal{D})$ and $\int_{E} v \mathrm{~d} s=0$, then

$$
\int_{\mathcal{D}} b v^{2} \mathrm{~d} x \mathrm{~d} y \leq \tilde{c}_{2}\left(h_{1}^{2} \int_{\mathcal{D}} v_{x}^{2} \mathrm{~d} x \mathrm{~d} y+\tilde{c}_{1}^{-1} h_{2}^{2} \int_{\mathcal{D}} b v_{y}^{2} \mathrm{~d} x \mathrm{~d} y\right) .
$$


Proof. Without loss of generality, we assume $\int_{0}^{h_{2}} v(0, y) \mathrm{d} y=0$. Then

$$
v(x, y)=v\left(0, y_{0}\right)+\int_{0}^{x} v_{x}\left(s, y_{0}\right) \mathrm{d} s+\int_{y_{0}}^{y} v_{y}(x, t) \mathrm{d} t .
$$

Integrating $y_{0}$ from 0 to $h_{2}$ and using $\int_{0}^{h_{2}} v\left(0, y_{0}\right) \mathrm{d} y_{0}=0$, we obtain

$$
v(x, y)=\frac{1}{h_{2}} \int_{0}^{h_{2}} \mathrm{~d} y_{0} \int_{0}^{x} v_{x}\left(s, y_{0}\right) \mathrm{d} s+\frac{1}{h_{2}} \int_{0}^{h_{2}} \mathrm{~d} y_{0} \int_{y_{0}}^{y} v_{y}(x, t) \mathrm{d} t .
$$

Squaring and then using the Cauchy-Schwarz inequality, we have

$$
\begin{aligned}
|v(x, y)|^{2} & \leq \frac{2}{h_{2}^{2}}\left(\int_{0}^{h_{2}} \mathrm{~d} y_{0} \int_{0}^{x}\left|v_{x}\left(s, y_{0}\right)\right| \mathrm{d} s\right)^{2}+\frac{2}{h_{2}^{2}}\left(\int_{0}^{h_{2}} \mathrm{~d} y_{0} \int_{0}^{y}\left|v_{y}(x, t)\right| \mathrm{d} t\right)^{2} \\
& \leq \frac{2}{h_{2}^{2}} h_{2} x \int_{0}^{h_{2}} \mathrm{~d} y_{0} \int_{0}^{h_{1}} v_{x}^{2}\left(s, y_{0}\right) \mathrm{d} s+\frac{2}{h_{2}^{2}} h_{2}^{2} y \int_{0}^{y} v_{y}^{2}(x, t) \mathrm{d} t \\
& \leq \frac{2 x}{h_{2}} \int_{\mathcal{D}} v_{x}^{2} \mathrm{~d} x \mathrm{~d} y+2 y \int_{0}^{h_{2}} v_{y}^{2}(x, t) \mathrm{d} t .
\end{aligned}
$$

Integrating over $\mathcal{D}$, we obtain

$$
\int_{\mathcal{D}}|v(x, y)|^{2} \mathrm{~d} x \mathrm{~d} y \leq h_{1}^{2} \int_{\mathcal{D}} v_{x}^{2} \mathrm{~d} x \mathrm{~d} y+h_{2}^{2} \int_{\mathcal{D}} v_{y}^{2} \mathrm{~d} x \mathrm{~d} y .
$$

This is the first part of the lemma.

We now prove the second part. Using $\epsilon(x) \leq 1$, we obtain from (4.1)

$$
\epsilon(x)|v(x, y)|^{2} \leq \frac{2 x}{h_{2}} \int_{\mathcal{D}} v_{x}^{2} \mathrm{~d} x \mathrm{~d} y+2 y \int_{0}^{h_{2}} \epsilon(x) v_{y}^{2}(x, t) \mathrm{d} t .
$$

Integrating the above inequality over $\mathcal{D}$ gives

$$
\int_{\mathcal{D}} \epsilon(x) v^{2} \mathrm{~d} x \mathrm{~d} y \leq\left(h_{1}^{2} \int_{\mathcal{D}} v_{x}^{2} \mathrm{~d} x \mathrm{~d} y+h_{2}^{2} \int_{\mathcal{D}} \epsilon(x) v_{y}^{2} \mathrm{~d} x \mathrm{~d} y\right) .
$$

Since $\tilde{c}_{1} \epsilon(x) \leq b(x, y) \leq \tilde{c}_{2} \epsilon(x)$, the second part of the lemma follows from the last inequality.

Using this lemma, we can prove the following error estimate for the nodal value interpolant.

Lemma 4.2. Let $\pi_{h}: C(\bar{\Omega}) \rightarrow M_{h}$ be the nodal value interpolation operator. Then

$$
\left\|\left(I-\pi_{h}\right) v\right\|_{A}^{2} \leq C h^{2} \int_{\Omega}\left(v_{x x}^{2}+v_{x y}^{2}+b v_{y y}^{2}\right) \mathrm{d} x \mathrm{~d} y, \quad \text { for all } v \in H^{2}(\Omega) .
$$

Proof. Let $\left(x_{i}, y_{j}\right)=(i h, j h)$, and let $\tau=\left[x_{i-1}, x_{i}\right] \times\left[y_{j-1}, y_{j}\right]$ be an arbitrary mesh rectangle. Let $E_{x}$ and $E_{y}$ be edges in the $x$ and $y$ directions, respectively. For the bilinear element, we have

$$
\int_{E_{x}}\left(v-\pi_{h} v\right)_{x} \mathrm{~d} x=\int_{E_{y}}\left(v-\pi_{h} v\right)_{y} \mathrm{~d} y=0 .
$$

Applying the first part of Lemma 4.1 to $\left(v-\pi_{h} v\right)_{x}$ and the second part of Lemma4.1 to $\left(v-\pi_{h} v\right)_{y}$ on each element $\tau$, we have

$$
\int_{\tau} a\left|\left(v-\pi_{h} v\right)_{x}\right|^{2} \mathrm{~d} x \mathrm{~d} y \leq a_{\max } h^{2} \int_{\tau}\left(\left|\left(v-\pi_{h} v\right)_{x x}\right|^{2}+\left|\left(v-\pi_{h} v\right)_{x y}\right|^{2}\right) \mathrm{d} x \mathrm{~d} y,
$$


and

$$
\int_{\tau} b\left|\left(v-\pi_{h} v\right)_{y}\right|^{2} \mathrm{~d} x \mathrm{~d} y \leq C h^{2} \int_{\tau}\left(\left|\left(v-\pi_{h} v\right)_{x y}\right|^{2}+b\left|\left(v-\pi_{h} v\right)_{y y}\right|^{2}\right) \mathrm{d} x \mathrm{~d} y .
$$

In the bilinear case, $\left(\pi_{h} v\right)_{x x}=\left(\pi_{h} v\right)_{y y}=0$ in $\tau$ and

$$
\begin{aligned}
\left\|\left(\pi_{h} v\right)_{x y}\right\|_{L^{2}(\tau)}^{2} & =h^{-2}\left|\left[v\left(x_{i}, y_{j}\right)-v\left(x_{i-1}, y_{j}\right)\right]-\left[v\left(x_{i}, y_{j-1}\right)-v\left(x_{i-1}, y_{j-1}\right)\right]\right|^{2} \\
& \leq\left\|v_{x y}\right\|_{L^{2}(\tau)}^{2} .
\end{aligned}
$$

Therefore,

$$
\begin{aligned}
\int_{\tau}[a \mid(v & \left.\left.-\pi_{h} v\right)\left._{x}\right|^{2}+b\left|\left(v-\pi_{h} v\right)_{y}\right|^{2}\right] \mathrm{d} x \mathrm{~d} y \\
& \leq C h^{2} \int_{\tau}\left(\left|\left(v-\pi_{h} v\right)_{x x}\right|^{2}+2\left|\left(v-\pi_{h} v\right)_{x y}\right|^{2}+b\left|\left(v-\pi_{h} v\right)_{y y}\right|^{2}\right) \mathrm{d} x \mathrm{~d} y \\
& \leq C h^{2} \int_{\tau}\left(\left|v_{x x}\right|^{2}+2\left|v_{x y}\right|^{2}+b\left|v_{y y}\right|^{2}\right) \mathrm{d} x \mathrm{~d} y .
\end{aligned}
$$

The result for bilinear elements follows by summing over $\tau$.

In the case of linear elements, we write $\tau=\tau^{+} \cup \tau^{-}$, where $\tau^{+}$and $\tau^{-}$are the two triangles on which $\pi_{h} v$ is linear. Let $\ell^{+}$be the linear function on $\tau$ which is equal to $\pi_{h} v$ on $\tau^{+}$, with $\ell^{-}$similarly defined. Applying the first part of Lemma 4.1 to $\left(v-\ell^{ \pm}\right)_{x}$ and the second part of Lemma 4.1 to $\left(v-\ell^{ \pm}\right)_{y}$ on $\tau$, we obtain

$$
\int_{\tau} a\left|\left(v-\ell^{ \pm}\right)_{x}\right|^{2} \mathrm{~d} x \mathrm{~d} y \leq a_{\max } h^{2} \int_{\tau}\left(\left|\left(v-\ell^{ \pm}\right)_{x x}\right|^{2}+\left|\left(v-\ell^{ \pm}\right)_{x y}\right|^{2}\right) \mathrm{d} x \mathrm{~d} y
$$

and

$$
\int_{\tau} b\left|\left(v-\ell^{ \pm}\right)_{y}\right|^{2} \mathrm{~d} x \mathrm{~d} y \leq C h^{2} \int_{\tau}\left(\left|\left(v-\ell^{ \pm}\right)_{x y}\right|^{2}+b\left|\left(v-\ell^{ \pm}\right)_{y y}\right|^{2}\right) \mathrm{d} x \mathrm{~d} y .
$$

Since $\ell^{ \pm}$is linear, its second derivatives all vanish in $\tau$, and hence we obtain

$$
\int_{\tau}\left(a\left|\left(v-\ell^{ \pm}\right)_{x}\right|^{2}+b\left|\left(v-\ell^{ \pm}\right)_{y}\right|^{2}\right) \mathrm{d} x \mathrm{~d} y \leq C h^{2} \int_{\tau}\left(\left|v_{x x}\right|^{2}+2\left|v_{x y}\right|^{2}+b\left|v_{y y}\right|^{2}\right) \mathrm{d} x \mathrm{~d} y .
$$

Consequently,

$$
\begin{aligned}
& \int_{\tau}[a \mid(v-\left.\left.\pi_{h} v\right)\left._{x}\right|^{2}+b\left|\left(v-\pi_{h} v\right)_{y}\right|^{2}\right] \mathrm{d} x \mathrm{~d} y \\
&= \int_{\tau^{+}}\left(a\left|\left(v-\ell^{+}\right)_{x}\right|^{2}+b\left|\left(v-\ell^{+}\right)_{y}\right|^{2}\right) \mathrm{d} x \mathrm{~d} y \\
& \quad+\int_{\tau^{-}}\left(a\left|\left(v-\ell^{-}\right)_{x}\right|^{2}+b\left|\left(v-\ell^{-}\right)_{y}\right|^{2}\right) \mathrm{d} x \mathrm{~d} y \\
& \leq C h^{2} \int_{\tau}\left(\left|v_{x x}\right|^{2}+2\left|v_{x y}\right|^{2}+b\left|v_{y y}\right|^{2}\right) \mathrm{d} x \mathrm{~d} y .
\end{aligned}
$$

The result now follows by summing over $\tau$.

The Galerkin projection has the following approximation properties.

Lemma 4.3. There is a constant $C_{1}$ such that, for $v \in H_{0}^{1}(\Omega) \cap H^{2}(\Omega)$,

$$
\left\|\left(I-P_{h}\right) v\right\|_{A}^{2} \leq C_{1} h^{2}\left(\frac{1}{b} \mathcal{L} v, \mathcal{L} v\right)
$$


and

$$
\left(b\left(I-P_{h}\right) v,\left(I-P_{h}\right) v\right) \leq C_{1} h^{2}\left\|\left(I-P_{h}\right) v\right\|_{A}^{2} .
$$

Proof. The first inequality is a consequence of Lemma 4.2 and Lemma 3.1, i.e.,

$$
\begin{aligned}
\left\|v-P_{h} v\right\|_{A}^{2} & \leq\left\|v-\pi_{h} v\right\|_{A}^{2} \\
& \leq C h^{2} \int_{\Omega}\left(v_{x x}^{2}+2 v_{x y}^{2}+b v_{y y}^{2}\right) \mathrm{d} x \mathrm{~d} y \\
& \leq C_{1} h^{2}\left(\frac{1}{b} \mathcal{L} v, \mathcal{L} v\right) .
\end{aligned}
$$

For the second we use a duality argument. Let $\mathcal{L} w=b\left(I-P_{h}\right) v$. Then

$$
\begin{aligned}
\left(b\left(I-P_{h}\right) v,\left(I-P_{h}\right) v\right) & =A\left(w, v-P_{h} v\right)=A\left(\left(I-P_{h}\right) w,\left(I-P_{h}\right) v\right) \\
& \leq\left\|\left(I-P_{h}\right) w\right\|_{A}\left\|\left(I-P_{h}\right) v\right\|_{A} \\
& \leq \sqrt{C_{1}} h\left(\frac{1}{b} \mathcal{L} w, \mathcal{L} w\right)^{1 / 2}\left\|\left(I-P_{h}\right) v\right\|_{A} \\
& =\sqrt{C_{1}} h\left(b\left(I-P_{h}\right) v,\left(I-P_{h}\right) v\right)^{1 / 2}\left\|\left(I-P_{h}\right) v\right\|_{A} .
\end{aligned}
$$

Cancelling the common factor and then squaring, we get the second inequality.

\section{The line Jacobi and Gauss-Seidel smoothers}

We consider only linear and bilinear elements. The partition of $\Omega$ and the finite element space $M_{h}$ are defined as in the previous section. To define the line Jacobi and the line Gauss-Seidel smoothers, we introduce a horizontal stripwise decomposition of $\Omega$ :

$$
\Omega=\bigcup \Omega_{j}, \quad \Omega_{j}=[(x, y) \in \Omega:(j-1) h<y<(j+1) h] .
$$

We partition the finite element space $M_{h}$ accordingly as

$$
M_{h}=\sum_{j=1}^{n-1} M_{h, j}, \quad \text { where } \quad M_{h, j}=\left[v \in M_{h}: v=0 \text { in } \Omega \backslash \Omega_{j}\right] .
$$

Note that for linear and bilinear elements this is a direct sum, i.e., the decomposition of $v \in M_{h}$ as $v=\sum_{j=1}^{n-1} v_{j}$ with $v_{j} \in M_{h, j}$ is unique. The operator $A_{h}: M_{h} \rightarrow M_{h}$ is defined by

$$
\left(A_{h} v, \phi\right)=A(v, \phi), \quad \text { for all } \quad \phi \in M_{h} .
$$

The operator $A_{h, j}: M_{h, j} \rightarrow M_{h, j}$, the "restriction" of $A_{h}$ to $M_{h, j}$, is defined similarly, i.e.,

$$
\left(A_{h, j} v, \phi\right)=A(v, \phi), \quad \text { for all } \phi \in M_{h, j} .
$$

We also need the projection $Q_{h, j}: M_{h} \rightarrow M_{h, j}$ with respect to the $L^{2}$ inner product $(\cdot, \cdot)$ and the projection $P_{h, j}: M_{h} \rightarrow M_{h, j}$ with respect to the inner product $A(\cdot, \cdot)$. Note that the relation $P_{h, j}=A_{h, j}^{-1} Q_{h, j} A_{h}$ holds.

The line Jacobi smoother $\mathcal{J}_{h}$ is defined by

$$
\partial_{h}=\sum_{j=1}^{n-1} A_{h, j}^{-1} Q_{h, j}
$$


The line Gauss-Seidel smoother $\mathcal{G}_{h}$ is defined by

$$
\mathcal{S}_{h}=\left[I-\left(I-P_{h, n-1}\right) \cdots\left(I-P_{h, 1}\right)\right] A_{h}^{-1}, \quad \text { where } P_{h, j}=A_{h, j}^{-1} Q_{h, j} A_{h} .
$$

Given $f \in M_{h}, \mathcal{G}_{h} f \in M_{h}$ can be computed as follows:

(i) Set $v_{0}=0$.

(ii) For $i=1, \ldots, n-1$ define

$$
v_{i}=v_{i-1}+A_{h, i}^{-1} Q_{h, i}\left(f-A_{h} v_{i-1}\right) .
$$

(iii) Set $\mathcal{G}_{h} f=v_{n-1}$.

To establish the smoothing property of the line Jacobi smoother we use the following characterization of $\mathcal{J}_{h}$ :

$$
\left(\mathfrak{J}_{h}^{-1} v, v\right) \equiv \sum_{j=1}^{n-1} A\left(v_{j}, v_{j}\right)
$$

where $\sum_{j} v_{j}=v$ with $v_{j} \in M_{h, j}\left(v_{j}\right.$ is unique). This result is trivial if we interpret the smoother, $\partial_{h}$, in (5.1) and (5.3) using a matrix-vector notation. A direct proof of (5.3) is also easy. We first note that $\left(\mathcal{J}_{h} v, v\right)=\sum_{j}\left(A_{h, j}^{-1} Q_{h, j} v, Q_{h, j} v\right)=0$ implies that $v=0$. Consequently $\mathcal{J}_{h}^{-1}$ exists. Let $v \in M_{h}$ and $v=\sum_{j} v_{j}$ with $v_{j} \in M_{h, j}$. Since the decomposition is unique, we have $v_{j}=A_{h, j}^{-1} Q_{h, j} \mathcal{J}_{h}^{-1} v$. Equality (5.3) now follows from a simple calculation using the formula for $v_{j}$.

A smoothing property of the line Jacobi operator is summarized in the following lemma.

Lemma 5.1. Let $M_{h}$ consist of piecewise linear or bilinear functions and let $\mathrm{J}_{h}$ be the line Jacobi smoother defined by (5.1). Then there is a constant $C_{2}$ such that

$$
\frac{1}{2} A(v, v) \leq\left(\mathcal{J}_{h}^{-1} v, v\right) \leq C_{2}\left[A(v, v)+\frac{1}{h^{2}}(b v, v)\right], \quad \text { for all } \quad v \in M_{h} .
$$

Proof. In either case, we write $v \in M_{h}$ as $v=\sum_{j} v_{j}$, with $v_{j} \in M_{h, j}$. Recall that this decomposition of $v$ is unique. Let $S_{j}=[(x, y) \in \Omega:(j-1) h \leq y \leq j h]$. Then by (5.3)

$$
\left(\mathcal{J}_{h}^{-1} v, v\right) \equiv \sum_{j=1}^{n-1} A\left(v_{j}, v_{j}\right)=\sum_{j=1}^{n}\left[A_{S_{j}}\left(v_{j-1}, v_{j-1}\right)+A_{S_{j}}\left(v_{j}, v_{j}\right)\right]
$$

On the strip $S_{j}$ we have $v=v_{j-1}+v_{j}\left(v_{0}=v_{n}=0\right)$. Thus

$$
A_{S_{j}}(v, v) \leq 2\left[A_{S_{j}}\left(v_{j-1}, v_{j-1}\right)+A_{S_{j}}\left(v_{j}, v_{j}\right)\right] .
$$

The first inequality follows by summing the above inequality from 1 to $n$.

We now prove the upper estimate for $\left(\mathcal{J}_{h}^{-1} v, v\right)$. In the bilinear case, note that

$$
v_{j}\left(x, y_{j}\right)=v\left(x, y_{j}\right) \quad \text { and } \quad v_{j}\left(x, y_{j \pm 1}\right)=0 .
$$


A simple calculation shows that

$$
\begin{aligned}
\int_{S_{j}}\left(\left|D_{x} v_{j-1}\right|^{2}\right. & \left.+\left|D_{x} v_{j}\right|^{2}\right) \mathrm{d} x \mathrm{~d} y \\
& =\frac{1}{3} \sum_{i=1}^{n-1}\left[\left|v\left(x_{i}, y_{j-1}\right)-v\left(x_{i-1}, y_{j-1}\right)\right|^{2}+\left|v\left(x_{i}, y_{j}\right)-v\left(x_{i-1}, y_{j}\right)\right|^{2}\right] \\
& \leq 2 \int_{S_{j}}\left|v_{x}\right|^{2} \mathrm{~d} x \mathrm{~d} y
\end{aligned}
$$

Consequently,

$$
\int_{S_{j}} a(x, y)\left(\left|D_{x} v_{j-1}\right|^{2}+\left|D_{x} v_{j}\right|^{2}\right) \mathrm{d} x \mathrm{~d} y \leq 2 \frac{a_{\max }}{a_{\min }} \int_{S_{j}} a(x, y)\left|v_{x}\right|^{2} \mathrm{~d} x \mathrm{~d} y .
$$

On the other hand, for $(x, y) \in S_{j}$,

$$
\left|D_{y} v_{j-1}(x, y)\right|^{2}+\left|D_{y} v_{j}(x, y)\right|^{2}=\frac{1}{h^{2}}\left(\left|v\left(x, y_{j-1}\right)\right|^{2}+\left|v\left(x, y_{j}\right)\right|^{2}\right) .
$$

Since $v_{y y}(x, \theta) \equiv 0$ for $\theta$ between $y_{j-1}$ and $y_{j}$, we have

$$
\begin{aligned}
\left|v\left(x, y_{j-1}\right)\right|^{2} & +\left|v\left(x, y_{j}\right)\right|^{2} \\
& =\left|v(x, y)+v_{y}(x, y)\left(y_{j-1}-y\right)\right|^{2}+\left|v(x, y)+v_{y}(x, y)\left(y_{j}-y\right)\right|^{2} \\
& \leq 4|v(x, y)|^{2}+2 h^{2}\left|v_{y}(x, y)\right|^{2},
\end{aligned}
$$

for all $(x, y) \in S_{j}$. Hence

$$
\int_{S_{j}} b\left(\left|D_{y} v_{j-1}\right|^{2}+\left|D_{y} v_{j}\right|^{2}\right) \mathrm{d} x \mathrm{~d} y \leq \frac{4}{h^{2}} \int_{S_{j}} b|v|^{2} \mathrm{~d} x \mathrm{~d} y+2 \int_{S_{j}} b\left|v_{y}\right|^{2} \mathrm{~d} x \mathrm{~d} y .
$$

Combining (5.4) and (5.5),

$$
\begin{aligned}
A_{S_{j}}\left(v_{j-1}, v_{j-1}\right) & +A_{S_{j}}\left(v_{j}, v_{j}\right) \\
& \leq \frac{4}{h^{2}} \int_{S_{j}} b v^{2} \mathrm{~d} x \mathrm{~d} y+2 \frac{a_{\max }}{a_{\min }} \int_{S_{j}}\left(a\left|v_{x}\right|^{2}+b\left|v_{y}\right|^{2}\right) \mathrm{d} x \mathrm{~d} y .
\end{aligned}
$$

Summing from 1 to $n$,

$$
\begin{aligned}
\left(\mathcal{J}_{h}^{-1} v, v\right) & \equiv \sum_{j=1}^{n}\left[A_{S_{j}}\left(v_{j-1}, v_{j-1}\right)+A_{S_{j}}\left(v_{j}, v_{j}\right)\right] \\
& \leq\left[2 \frac{a_{\max }}{a_{\min }} A(v, v)+\frac{4}{h^{2}}(b v, v)\right] .
\end{aligned}
$$

This proves the second inequality for the bilinear case.

The proof for the case of linear elements is similar. We write $\tau=\tau^{+} \cup \tau^{-}$. Then

$$
D_{x} v_{j-1}=0, D_{x} v_{j}=D_{x} v \text { on } \tau^{+} \text {and } D_{x} v_{j}=0, D_{x} v_{j-1}=D_{x} v \text { on } \tau^{-} \text {. }
$$

Therefore

$$
\int_{\tau} a\left(\left|D_{x} v_{j-1}\right|^{2}+\left|D_{x} v_{j}\right|^{2}\right) \mathrm{d} x \mathrm{~d} y=\int_{\tau} a\left|D_{x} v\right|^{2} \mathrm{~d} x \mathrm{~d} y .
$$

On the other hand,

$$
\left|D_{y} v_{j-1}\right|^{2}+\left|D_{y} v_{j}\right|^{2}=\frac{1}{h^{2}}\left(\left|v\left(x_{i-1}, y_{j-1}\right)\right|^{2}+\left|v\left(x_{i-1}, y_{j}\right)\right|^{2}\right) \quad \text { in } \tau^{+}
$$


and

$$
\left|D_{y} v_{j-1}\right|^{2}+\left|D_{y} v_{j}\right|^{2}=\frac{1}{h^{2}}\left(\left|v\left(x_{i}, y_{j-1}\right)\right|^{2}+\left|v\left(x_{i}, y_{j}\right)\right|^{2}\right) \quad \text { in } \tau^{-} .
$$

Since all the second derivatives of $v$ vanish on $\tau^{ \pm}$, we have

$$
\begin{aligned}
\left|v\left(x_{i-1}, y_{j-1}\right)\right|^{2}+ & \left|v\left(x_{i-1}, y_{j}\right)\right|^{2} \\
= & \left|v(x, y)+\nabla v(x, y) \cdot\left(x_{i-1}-x, y_{j-1}-y\right)\right|^{2} \\
& +\left|v(x, y)+\nabla v(x, y) \cdot\left(x_{i-1}-x, y_{j}-y\right)\right|^{2} \\
\leq & 4|v(x, y)|^{2}+6 h^{2}|\nabla v(x, y)|^{2}, \text { for all }(x, y) \in \tau^{+} .
\end{aligned}
$$

A similar estimate holds for $\left|v\left(x_{i}, y_{j-1}\right)\right|^{2}+\left|v\left(x_{i}, y_{j}\right)\right|^{2}$. Hence,

$$
\begin{aligned}
\int_{\tau} b\left(\left|D_{y} v_{j-1}\right|^{2}+\right. & \left.\left|D_{y} v_{j}\right|^{2}\right) \mathrm{d} x \mathrm{~d} y \\
= & \frac{1}{h^{2}}\left[\int_{\tau^{+}} b\left(\left|v\left(x_{i-1}, y_{j-1}\right)\right|^{2}+\left|v\left(x_{i-1}, y_{j}\right)\right|^{2}\right) \mathrm{d} x \mathrm{~d} y\right. \\
& \left.\quad+\int_{\tau^{-}} b\left(\left|v\left(x_{i}, y_{j-1}\right)\right|^{2}+\left|v\left(x_{i}, y_{j}\right)\right|^{2}\right) \mathrm{d} x \mathrm{~d} y\right] \\
\leq & \frac{4}{h^{2}} \int_{\tau} b v^{2} \mathrm{~d} x \mathrm{~d} y+6 \int b|\nabla v|^{2} \mathrm{~d} x \mathrm{~d} y
\end{aligned}
$$

Combining the estimates for $\int_{\tau} a\left(\left|D_{x} v_{j-1}\right|^{2}+\left|D_{x} v_{j}\right|^{2}\right)$ and $\int_{\tau} b\left(\left|D_{y} v_{j-1}\right|^{2}+\left|D_{y} v_{j}\right|^{2}\right)$ we obtain

$$
A_{S_{j}}\left(v_{j-1}, v_{j-1}\right)+A_{S_{j}}\left(v_{j}, v_{j}\right) \leq \frac{4}{h^{2}} \int_{S_{j}} b v^{2} \mathrm{~d} x \mathrm{~d} y+\int_{S_{j}}\left[(1+6 b) v_{x}^{2}+6 b v_{y}^{2}\right] \mathrm{d} x \mathrm{~d} y
$$

The rest of the proof is identical to that for the bilinear elements.

We formulate the smoothing property of the line Gauss-Seidel smoother in the next lemma.

Lemma 5.2. Let $M_{h}$ consist of piecewise linear or bilinear functions and let $\mathcal{G}_{h}$ be the line Gauss-Seidel smoother defined in (5.2). Then there is a constant $C_{2}$ such that

$$
A(v, v) \leq\left(\overline{\mathcal{G}}_{h}^{-1} v, v\right) \leq C_{2}\left[A(v, v)+\frac{1}{h^{2}}(b v, v)\right], \quad \text { for all } \quad v \in M_{h},
$$

where $\overline{\mathcal{G}}_{h} \equiv \mathcal{G}_{h}+\mathcal{G}_{h}^{t}-\mathcal{G}_{h}^{t} A_{h} \mathcal{G}_{h}$ is the symmetric line Gauss-Seidel smoother.

Proof. By the definition of $\overline{\mathcal{G}}_{h}$, we have

$$
0 \leq A\left(\left(I-\mathcal{G}_{h} A_{h}\right) v,\left(I-\mathcal{G}_{h} A_{h}\right) v\right)=A\left(\left(I-\overline{\mathcal{G}}_{h} A_{h}\right) v, v\right) \quad \text { for all } \quad v \in M_{h} .
$$

This implies the lower estimate. The upper estimate follows from the inequality

$$
A\left(\mathcal{J}_{h} A_{h} v, v\right) \leq C A\left(\overline{\mathcal{G}}_{h} A_{h} v, v\right)
$$

and the upper estimate for the line Jacobi smoother in Lemma 5.1.

Remark 5.1. In the proof of Lemma 5.1, we did not make use of (1.4). With minor modifications, we can prove that the results in Lemmas 5.1 and 5.2 hold for general polynomial elements, provided that (1.4) holds. 


\section{Multigrid COnvergence Estimate}

We now establish a uniform convergence result for the V-cycle multigrid Algorithm 2.1. For simplicity, we only consider linear and bilinear elements. We introduce an initial triangulation $\mathcal{T}_{1}$ of $\Omega$ by partitioning $\Omega$ into four smaller equal squares. For linear elements, each square is further decomposed into two triangles by linking the lower-left and upper-right vertices. Let $\left\{\mathcal{T}_{k}\right\}$ be a family of triangulations of $\Omega$, where $\mathcal{T}_{k}$ is obtained from $\mathcal{T}_{k-1}$ by a halving strategy. Let $\left\{M_{k}\right\}$ be the corresponding family of linear or bilinear finite element spaces defined with respect to $\left\{\mathcal{T}_{k}\right\}$. Denote by $\mathcal{J}_{h_{k}}$ and $\mathcal{G}_{h_{k}}$ respectively the line Jacobi and the line Gauss-Seidel operators on $M_{k}$.

Theorem 6.1. Let $R_{k}=\eta \mathcal{J}_{h_{k}}$ with $0<\eta<1$, or $R_{k}=\mathcal{G}_{h_{k}}$. Then there is a positive number $\delta$ with $\delta<1$, independent of $k$, such that the multigrid algorithm defined in Algorithm 2.1 satisfies

$$
0 \leq A\left(\left(I-B_{k} A_{k}\right) v, v\right) \leq \delta A(v, v), \quad \text { for all } \quad v \in M_{k}
$$

Proof. We first show the result for $R_{k}=\eta \mathcal{J}_{h_{k}}$. By Lemma 5.1] the spectrum $\sigma\left(R_{k} A_{k}\right) \subset(0,2 \eta]$. Thus $\sigma\left(K_{k}\right)=\sigma\left(I-R_{k} A_{k}\right) \subset[-\theta, 1)$ with $\theta=2 \eta-1<1$. In view of Lemma 2.1, we only need to prove that (2.4) holds for $R_{k}=\eta \mathcal{J}_{h_{k}}$. It follows from Lemma 4.3 that

$$
\frac{1}{h^{2}}\left(b(x, y)\left(I-P_{k-1}\right) v,\left(I-P_{k-1}\right) v\right) \leq C_{1} A\left(\left(I-P_{k-1}\right) v,\left(I-P_{k-1}\right) v\right) .
$$

By Lemma 5.1 the weighted line Jacobi smoother, $R_{k}$, satisfies

$$
\left(R_{k}^{-1} \phi, \phi\right) \leq C_{2}\left[\left(A_{k} \phi, \phi\right)+\frac{1}{h_{k}^{2}}(b(x, y) \phi, \phi)\right], \quad \text { for all } \quad \phi \in M_{k} .
$$

Applying the smoothing property of $R_{k}$ to $\phi=\left(I-P_{k-1}\right) v$ and using the approximation property of $P_{k-1}$, we obtain, with $C_{M}=C_{2}\left(1+C_{1}\right)$,

$$
\left(R_{k}^{-1}\left(I-P_{k-1}\right) v,\left(I-P_{k-1}\right) v\right) \leq C_{M} A\left(\left(I-P_{k-1}\right) v,\left(I-P_{k-1}\right) v\right) .
$$

We have thus proved (2.4), and hence the theorem, for the case $R_{k}=\eta \mathcal{J}_{h_{k}}$.

The proof for the case $R_{k}=\mathcal{G}_{h_{k}}$ is analogous. We use Lemma 2.2 in place of Lemma 2.1 and use the smoothing property of the line Gauss-Seidel in Lemma 5.2 instead of Lemma 5.1

Since $B_{k} A_{k}$ is symmetric in the energy inner product $A(\cdot, \cdot)$, Theorem 6.1 implies that $\left\|\left(I-B_{k} A_{k}\right) v\right\|_{A} \leq \delta\|v\|_{A}$ for all $v \in M_{k}$. Consequently, the error operator of the multigrid iteration (2.1) is a uniform contraction in the $\|\cdot\|_{A}$ norm and the iterates defined in (2.1) satisfy

$$
\left\|u^{m}-u\right\|_{A} \leq \delta^{m}\left\|u^{m}-u\right\|_{A} .
$$

Remark 6.1. It is desirable to avoid the condition in (1.4) in our theory. However, it is not clear that this is possible even in the case when $b(x, y)$ is uniformly bounded from above and below.

Remark 6.2. Note that if $b(x, y) \approx h_{k}^{2}$, then the error operators, $I-\eta \mathcal{J}_{k} A_{k}$ and $I-\mathcal{G}_{k}$, corresponding to the line Jacobi and the line Gauss-Seidel methods, are already uniform contractions on $M_{k}$. 
Remark 6.3. Our analysis remains valid for other polynomial finite elements as well. The approximation property is a consequence of the fact that the linear elements are a subspace of these higher order elements and the smoothing property can be established in a way similar to that of the linear element.

\section{Matrix-VeCtor implementation}

We now discuss briefly the implementation of the multigrid algorithm using a matrix-vector notation. Let $\left\{\phi_{k}^{i}\right\}, i=1, \ldots, N_{k}$, be the nodal basis of $M_{k}$. Then each function $v$ in $M_{k}$ is associated with two vectors, its "coefficient vector" $\tilde{v}$ and its "dual vector" $\underset{\sim}{v}$. The components of the coefficient vector $\tilde{v}$ consist of the coefficients of $v$ with respect to the basis $\left\{\phi_{k}^{i}\right\}$, and the dual vector, defined by $\underset{\sim}{v}=\left[\left(v, \phi_{k}^{i}\right)\right]$, represents the action of $v$ on the basis $\left\{\phi_{k}^{i}\right\}$. Corresponding to the operator $A_{k}$ is the stiffness matrix $\underset{\approx}{A_{k}}=\left[A\left(\phi_{k}^{i}, \phi_{k}^{j}\right)\right]$. Using this notation, the finite element equation $A_{k} u_{k}=f_{k}$ can be written as a linear system of equations:

$$
\underset{\sim}{A} A_{k} \tilde{u}_{k}=\underset{\sim}{f_{k}} \text {. }
$$

To define the multigrid algorithm in terms of vectors and matrices, we introduce, for each smoother $R_{k}$, a smoothing matrix $\widetilde{R}_{k}$ satisfying

$$
\widetilde{R_{k} f_{k}}=\widetilde{\widetilde{R}}_{k} f_{\sim}, \quad \text { for all } f_{k} \in M_{k} \text {. }
$$

It is easy to check that the smoothing matrices corresponding to $R_{k}=\eta \mathcal{J}_{h_{k}}$ and $R_{k}=\mathcal{G}_{h_{k}}$ are just the block diagonal and lower block triangular parts of $A_{k}$.

Since $M_{k-1} \subset M_{k}$, the basis functions of $M_{k-1}$ can be expressed in $\widetilde{\text { terms of }}$ those of $M_{k}$, i.e.,

$$
\phi_{k-1}^{i}=\sum_{j=1}^{N_{k}} \alpha_{i j}^{k} \phi_{k}^{j} .
$$

The "interpolation matrix" and the "prolongation matrix" are given respectively by $\Pi_{k-1}=\left[\alpha_{i j}^{k}\right]$ and $\Pi_{k-1}^{t}$.

With the above notation, the coefficient vectors, $\tilde{u}^{m}$, of the multigrid iterates $u^{m}$ defined in (2.1) can be computed by

$$
\tilde{u}^{m+1}=\tilde{u}^{m}+\widetilde{\widetilde{B}}_{k}\left(\underset{\sim}{f_{k}}-\underset{\approx}{A} \tilde{u}^{m} \tilde{u}^{m}\right), \quad m=0,1, \ldots,
$$

where $\widetilde{B}_{k}$ is defined recursively by the following algorithm:

Algorithm 7.1 (Matrix-vector form). Set $\widetilde{\widetilde{B}}_{1}=\underset{\approx}{A_{1}^{-1}}$. Assume that $\widetilde{\widetilde{B}}_{k-1}$ has been defined. Define $\widetilde{B}_{k}: R^{N_{k}} \rightarrow R^{N_{k}}$ as follows.

(1) Pre-smoothing: Set $\tilde{v}^{\prime}=\tilde{v}+\widetilde{\widetilde{R}}_{k}^{t}\left(\underset{\sim}{f_{k}}-\underset{\approx}{A}{ }_{k} \tilde{v}\right)$.

(2) Correction: Define $\tilde{v}^{\prime \prime}=\tilde{v}^{\prime}+\Pi_{k-1}^{t} \tilde{q}(\tilde{v}=0)$, where

$$
\tilde{q}=\widetilde{\widetilde{B}}_{k-1} \Pi_{k-1}\left(f_{\sim} k-\underset{\approx}{A_{k}} \tilde{v}^{\prime}\right)
$$

(3) Post-smoothing: Set $\widetilde{\widetilde{B}}_{k} f_{\sim}=\tilde{v}^{\prime \prime}+\widetilde{\widetilde{R}}_{k}\left(\underset{\sim}{f_{k}}-\underset{\approx}{A_{k}} \tilde{v}^{\prime \prime}\right)$.

It is straightforward to check that if $\widetilde{\widetilde{R}}_{k}$ and $R_{k}$ are related by (7.1), then

$$
\widetilde{B_{k} f_{k}}=\widetilde{\widetilde{B}}_{k} f_{\sim}, \quad \text { for all } \underset{\sim}{f_{k} \in M_{k}} \text {. }
$$


Therefore, Algorithms 7.1 and 2.1 are the same and $\tilde{u}^{m}$ defined by (7.2) is the coefficient vector of $u^{m}$ defined by (2.1), provided that $f_{k}$ is the dual vector of $f_{k}$ and $\tilde{u}^{0}$ is chosen to be the coefficient vector of $u^{0}$.

\section{REFERENCES}

1. I. Babuška and A. K. Aziz, On the angle condition in the finite element method, SIAM J. Numer. Anal. 13 (1976), no. 2, 214-226. MR 56:13700]

2. R. E. Bank, J. Mandel, and S. McCormick, Variational multigrid theory, Multigrid Methods (Philadelphia, PA) (S. McCormick, ed.), SIAM, 1987, pp. 131-178. MR 89m:65004

3. D. Braess and W. Hackbusch, A new convergence proof for the multigrid method including the V-cycle, SIAM J. Numer. Anal. 20 (1983), 967-975. MR 85h:65233

4. J. H. Bramble and J. E. Pasciak, New convergence estimates for multigrid algorithms, Math. Comp. 49 (1987), 311-329. MR 89b:65234]

5. — New estimates for multilevel algorithms including the V-cycle, Math. Comp 60 (1993), 447-471. MR 94a:65064

6. _ Uniform convergence estimates for multigrid $V$-cycle algorithms with less than full elliptic regularity, Domain Decomposition Methods in Science and Engineering: The Sixth International Conference on Domain Decomposition (Alfio Quarteroni, Yuri A. Kuznetsov, Jacques Périaux, and Olof B. Widlund, eds.), Contemporary Mathematics, vol. 157, 1994, Held in Como, Italy, June 15-19, 1992, pp. 7-26. MR 95f:65202

7. J. H. Bramble, J. E. Pasciak, J. Wang, and J. Xu, Convergence estimates for multigrid algorithms without regularity assumptions, Math. Comp. 57 (1991), 23-45. MR 91m:65158

8. M. Griebel and P. Oswald, Tensor-product-type subspace splittings and multilevel methods for anisotropic problems., Adv. Comput. Math. 4 (1995), 171-206. MR 96e:65069

9. W. Hackbusch, Multi-grid methods and applications, Springer series in computational mathematics, vol. 4, Springer-Verlag, Berlin, New York, 1985. MR 87e:65082

10. P.W. Hemker, Multigrid methods for problems with a small parameter in the highest derivative, Numerical Analysis. Proceedings Dundee 1983. Lecture Notes in Mathematics, 1066 (Springer, Berlin Heidelberg New York) (D.F. Griffiths, ed.), 1984, pp. 106-121. MR 85i:65158

11. N. Neuss, V-cycle convergence with unsymmetric smoothers and application to an anisotropic model problem, SIAM J. Numer. Anal. 35 (1998), 1201-1212. MR 99d:65109

12. R. Stevenson, New estimates of the contraction number of V-cycle multi-grid with applications to anisotropic equations, Incomplete Decompositions, Proceedings of the Eighth GAMM Seminar. Notes on Numerical Fluid Mechanics, Volume 41 (Wolfgan Hackbusch and Gabriel Wittum, eds.), 1993, pp. 159-167. MR 94d:65064

13. Robustness of multi-grid applied to anisotropic equations on convex domains and domains with re-entrant corners, Numer. Math. 66 (1993), 373-398. MR 94i:65047]

14. _ Modified ILU as a smoother, Numer. Math. 68 (1994), 295-309. MR 95d:65031

15. _ Robust multi-grid with 7-point ILU smoothing, Multigrid Methods IV, Proceedings of the Fourth European Multigrid Conference, Amsterdam (P.W. Hemker and Pieter D. Wesseling, eds.), Birkhäuser, 1994, pp. 295-307. MR 95g:65173

16. _ Robustness of the additive and multiplicative frequency decomposition multi-level method, Computing 54 (1995), 331-346. MR 96c:65194

17. G. Wittum, Linear iterations as smoothers in multigrid methods: theory with applications to incomplete decompositions, IMPACT Comput. Sci. Eng. 1 (1989), 180-215.

18. _ On the robustness of ILU smoothing, SIAM J. Sci. Stat. Comput. 10(4) (1989), 699-717. MR 90m:65208

19. J. Xu, Iterative methods by space decomposition and subspace correction, SIAM Review 34 (1992), no. 4, 581-613. MR 93k:65029

20. H. Yserentant, Old and new convergence proofs for multigrid methods, Acta Numerica (1993), 285-326. MR 94i:65128

Department of Mathematics, Texas A\&M University, College Station, TX 77843

E-mail address: bramble@math.tamu.edu

$U R L:$ http://www. math.tamu.edu/ ${ }^{\text {bramble }}$

Department of Mathematics, Texas A\&M University, College Station, TX 77843

E-mail address: xzhang@math.tamu.edu

$U R L$ : http://www. math.tamu.edu/ xzhang 